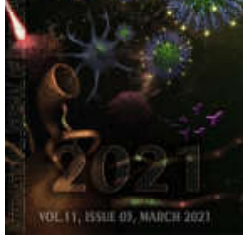

\title{
PREVALÊNCIA DE DOENÇAS PSÍQUICAS E METODOLOGIAS TERAPÊUTICAS NA POPULAÇÃO DE ÉRICO CARDOSO - BAHIA, BRASIL
}

\author{
1Bárbara Soares Aguiar, ${ }^{1}$ Ivan Gilson Silva Moura, ${ }^{1}$ Mariane Costa Santos de Tavares, ${ }^{1}$ Andressa \\ Dias de Andrade Fontes Amorim, ${ }^{1}$ Karina Pereira Neris and ${ }^{2}$ Caroline Braga Palacio
}

\author{
1Faculdades Santo Agostinho de Vitória da Conquista - BA \\ 2Médica. DSEI Alto Rio Solimões, Pólo Base Belém do Solimões
}

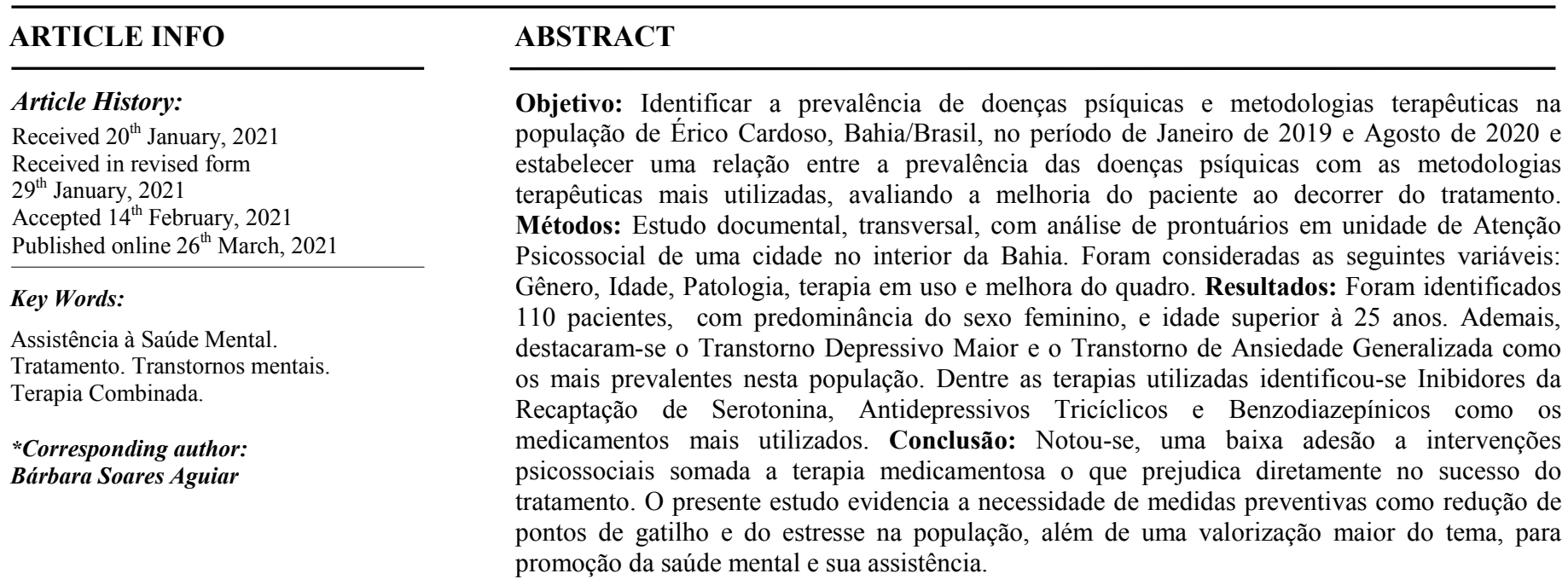

Copyright $\odot$ 2021, Bárbara Soares Aguiar et al. This is an open access article distributed under the Creative Commons Attribution License, which permits unrestricted use, distribution, and reproduction in any medium, provided the original work is properly cited.

Citation: Bárbara Soares Aguiar, Ivan Gilson Silva Moura, Mariane Costa Santos de Tavares, Andressa Dias de Andrade Fontes Amorim, Karina Pereira Neris and Caroline Braga Palacio 2021. "Prevalência de doenças psíquicas e metodologias terapêuticas na população de Érico Cardoso - Bahia, Brasil”, International Journal of Development Research, 11, (03), 45396-45399.

\section{INTRODUÇÃO}

Cada parte do cérebro é composto por uma função especifica, que se afetada pode alterar o estilo de vida da pessoa, por exemplo, o sistema límbico que é composto por várias estruturas, tais como: amígdala, hipotálamo, hipocampo, corpo caloso e muito outros, que no qual gerencia respostas fisiológicas para estímulos emocionais, relacionando-se com à memória, atenção, atitudes instintivas, personalidade e comportamento. Entretanto, se alguma dessas estruturas forem afetadas, altera concomitantemente a resposta na função específica da área acometida, ou seja, da resposta fisiológica passa a ser patológica (Sadock, Sadock, \& Ruiz, 2017). A literatura aponta que os transtornos mentais tem se tornado cada vez mais frequentes na população, contribuindo para uma grande carga de incapacidade, adoecimento e decréscimo da qualidade de vida (Smolen \& Araújo, 2017). Em estudo realizado no município de Ibicuí - Bahia/Brasil, com 310 idosos verificou-se uma prevalência de transtornos mentais comuns (TMC) de 55,8\%, gerando grande impacto na funcionalidade, e condição de saúde dos indivíduos afetados (Silva, et al., 2018). A Organização Mundial de Saúde (OMS, 2019) afirma que a maioria dos casos de saúde mental entre os jovens não é diagnosticada nem tratada. A ansiedade e depressão estão entre as principais causas de adoecimento e acometimento na saúde dos jovens, por conseguinte, o suicídio se tornou a segunda maior causa de morte entre os indivíduos entre 15 a 29 anos (OMS, 2019). A depressão em si é caracterizada por um estado de tristeza e isolamento, as vezes pode-se ser normal em pessoas sadias que passaram por perdas significativas, como luto de alguém próximo, perda de algum trabalho ou separação, mas também pode estar relacionada com um distúrbio psíquico, com tristeza ou humor disfórico e uma variedade de sintomas físicos e comportamentais (Basso \& Wainer, 2011). Os TMC, além de extremamente prevalentes na população brasileira são referidos como problemas de difícil manejo, sobretudo pelo elevado preconceito existente, baixa adesão ao tratamento, bem como acessibilidade prejudicada aos serviços de saúde. Outro problema existente é a abordagem centrada em tratamentos farmacológicos, sem que haja compartilhamento de técnicas terapêuticas complementares e/ou associadas (Soares, et al., 2021). 
Destaca-se, então, a importância do tratamento não farmacológico pela Equipe de Saúde da Família e a necessidade de capacitá-los para abordagem dos pacientes psiquiátricos pois eles costumam ser despercebidos principalmente no ambiente de atenção primária. Ratifica-se, que uma das problemáticas neste nível de atenção é a incapacidade dos profissionais de saúde de diagnosticar e tratar as enfermidades mentais presentes nos pacientes no decorrer do trabalho diário(Amaral, Torrenté, Torrenté, \& Moreira, 2018). Levando-se em consideração a relevância epidemiológica dos transtornos mentais, bem como a dificuldade do manejo destes, o objetivo do estudo aqui relatado foi identificar a prevalência de doenças psíquicas e metodologias terapêuticas na população de Érico Cardoso, Bahia, no período de Janeiro de 2019 e Agosto de 2020 e estabelecer uma relação entre a prevalência das doenças psíquicas com as metodologias terapêuticas mais utilizadas, avaliando a melhoria do paciente ao decorrer do tratamento.

\section{MATERIAIS E MÉTODOS}

Estudo exploratório, descritivo, transversal, em que analisaram os prontuários referente as doenças psíquicas em uma unidade de atenção psicossocial de uma cidade do interior da Bahia. Foi utilizado um instrumento estruturado para coleta de dados contendo variáveis relacionadas com Gênero, Idade, Patologia com a Classificação Internacional de Doenças (CID), o medicamento em uso, se está em associação a Terapia Cognitiva Comportamental ou outro método de intervenção psicossocial, tempo de tratamento, continuidade com o tratamento e a melhoria do paciente. A análise descritiva dos dados, com levantamento de gráficos, cálculo das médias e somatórios foram tabuladas através do Microsoft Excel ${ }^{\circledR}$. O projeto foi submetido e aprovado pelo Comitê de Ética em Pesquisa (CEP) da Faculdade de Enfermagem da Universidade Federal de Pelotas (UFPEL), com o seguinte número do parecer do protocolo de aprovação: 4.100.620.

\section{RESULTADOS}

Foram identificados 110 pacientes com doenças psíquicas no período entre Janeiro de 2019 e Agosto de 2020. Deste modo, todos os prontuários foram analisados integralmente não exclusão. Assim, foi percebido que a maioria era do sexo feminino $(n=66)$ representando o percentual de $60 \%$ e $40 \%$ do sexo masculino. A maioria dos pacientes tinham mais de 25 anos, sendo 74 pacientes do total $(67,3 \%)$. Enquanto apenas 13 pessoas tinham menos de 15 anos (11,8\%), 21 pessoas entre 15 e 25 anos $(19 \%)$ e 2 pacientes não foram identificados o a idade no prontuário $(1,8 \%)$. Abordando sobre a prevalência das doenças psíquicas nesta população $(n=110)$, observase que 19 foram diagnosticados com Transtorno Depressivo Recorrente (17,27\%), classificado como F33 no CID 10. Dezoito pacientes $(16,36 \%)$ foram diagnosticados com Transtorno de Ansiedade Generalizada, classificado como F41 no CID 10. Doze pacientes $(10,91 \%)$ foram diagnosticados com esquizofrenia, classificado como F20 no CID 10. Dez pacientes com Transtorno Depressivo Moderado (9,09\%), classificado como F32.1 no CID 10, sete pacientes com Transtorno Bipolar $(6,36 \%)$, outros sete com Distúrbio Intelectual (DI), antigo Retardo Mental no DSM IV, $(6,3 \%)$, classificado como F71 no CID 10. Houve ainda outros transtornos, com 'n' entre 1 e 2, tais como: Distúrbios da atividade de atenção (F90 no CID 10), Epilepsia (G40 no CID 10), Reação Aguda ao Stress (F43.0 no Cid 10), Transtorno de Pânico (F41 no CID 10), Transtorno Obsessivo Compulsivo (F42 no CID 10), Transtorno de Personalidade (F60 no Cid 10), automutilação (Z91.5 no CID 10), totalizando $26,36 \%$ do total da amostra (Gráfico 1 ). Em relação à adesão ao tratamento medicamentoso proposto, verificou-se que do total de pacientes analisados $(n=110) 37$ aderiram adequadamente ao tratamento, representando uma taxa de adesão de 33,64\%. A participação em sessões de psicoterapia foi identificado em 16 pacientes $(14,55 \%)$. Analisando a relação entre pacientes aderentes ao tratamento e participação em psicoterapia, tem-se que do total de pacientes aderentes $(n=37), 43,24 \%$ possuíam também intervenção psicoterápica.

\section{Diagnósticos de Transtornos mentais (\%)}

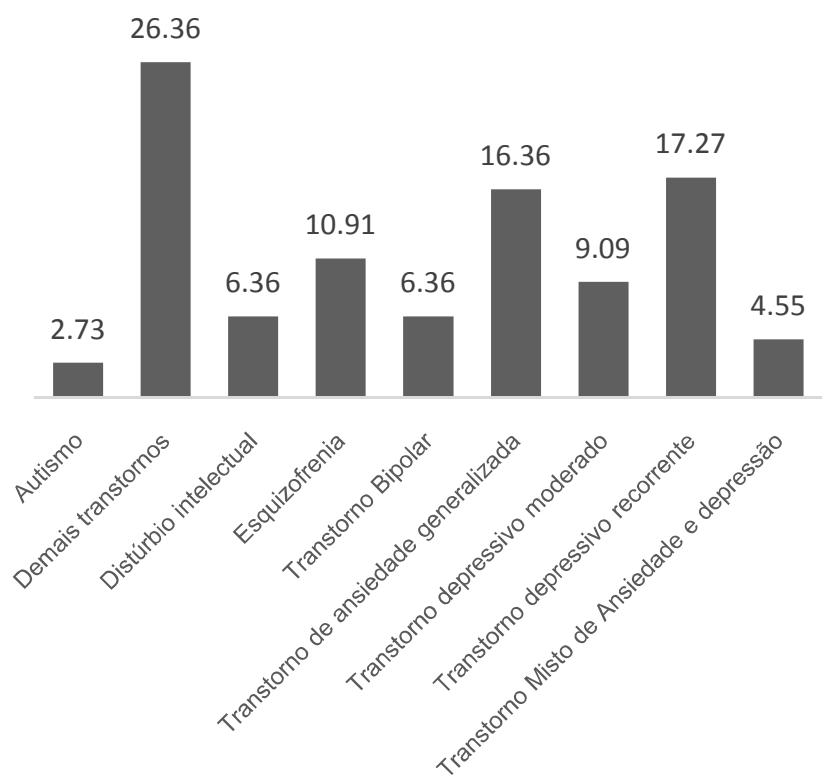

Fonte: Dados coletados pela pesquisa (2020).

\section{Gráfico 1: Diagnósticos identificados na amostra do estudo. Érico Cardoso, Bahia, 2020}

Nos pacientes com transtornos depressivos, incluindo transtorno depressivo moderado e recorrente $(n=29)$, verificou-se a participação em psicoterapia por $61,58 \%$ destes. Outros distúrbios como transtorno bipolar (50\%), distúrbio intelectual $(50 \%)$ e esquizofrenia $(40 \%)$ a participação em psicoterapia também foi bastante significativa (Tabela 1). Abordando sobre a prevalência das doenças psíquicas nesta população, observa-se que dos 110 pacientes, 19 foram diagnosticados com Transtorno Depressivo Recorrente (17\%), classificado como F33 no CID 10. Dentre eles, 13 pacientes utilizam politerapia e apenas 6 monoterapia sendo Amitripitilina (Antidepressivo Triciclico) e Risperidona (antipsicótico atípico) os medicamentos mais utilizados, havendo também Fluoxetina (Antidepressivo Inibidor de Recaptação de Serotonina), Escitalopram (Inibidor Seletivo da Recaptação de Serotonina) e Sertralina (Inibidor de Recaptação de Serotonina) como forma terapêutica. Além disso, 7 pacientes precisam de Clonazepam para tratamento da insônia ou controle de outros sintomas apresentados nas crises depressivas. Dos 19 pacientes acima citados, 10 deram continuidade a terapia e por isso mantiveram-se estáveis e apenas 6 destes fazem acompanhamento psicoterápico. se que do total 18 pacientes foram diagnosticados com Transtorno de Ansiedade Generalizada (16,3\%), classificado como F41 no CID 10. Dentre eles, 11 pacientes utilizam monoterapia e apenas 8 politerapia que em sua maioria é associado ao Clonazepam (Benzodiazepínicos) sendo Fluoxetina, Escitalopram os medicamentos mais utilizados, no entanto, prescreveram-se também, Amitripitilina, Nortripitilina (antidepressivo tricíclico) e Venfalaxina (Inibidores seletivos da receptação da Serotonina e Noradrenalina) para esses pacientes. Além desses transtornos acima citados, observou-se também 12 pacientes com Esquizofrenia (10,9\%), classificado como F20 no CID 10. Dentre eles, a maioria (7 pacientes) utilizam Biperidona ou Risperidona, além do mais dos 12 pacientes 10 fazem politerapia com Depakene (anticonvulsivante) ou Clonazepam, somando a estes foi visto também Quetiapina (Antipsicóticos Atípicos) e Haldol (Neuroléptico) como medicamentos prescritos. Contudo, observando os pacientes esquizofrênicos apenas 5 continuaram o tratamento sendo vista uma evolução positiva, mas apenas 2 pacientes fazem acompanhamento no Centro de Atenção Psicossocial (CAPS). observado também, 10 pacientes com Transtorno Depressivo Moderado (9\%), classificado como F32.1 no CID 10. 
Tabela 1. Relação entre diagnóstico, adesão ao tratamento e participação em psicoterapia. Érico Cardoso, Bahia, 2020

\begin{tabular}{llll}
\hline Diagnóstico & Adesão & Psicoterapia & Adesão x Psicoterapia (\%) \\
\hline Transtorno depressivo moderado & 3 & 3 & 100 \\
Transtorno depressivo recorrente & 10 & 6 & 60 \\
Transtorno Bipolar & 4 & 2 & 50 \\
Transtorno de ansiedade generalizada & 3 & 0 & 0 \\
Esquizofrenia & 5 & 2 & 40 \\
Distúrbio intelectual & 2 & 1 & 50 \\
Transtorno Misto de Ansiedade e depressão & 2 & 0 & 0 \\
Autismo & 3 & 0 & 0 \\
Demais transtornos & 5 & 2 & 40 \\
Total & 37 & 16 & 43,24 \\
\hline Fonte: Dados coletados pela pesquisa (2020). &
\end{tabular}

Desses 10 pacientes 6 usam Nortripitilina e 6 Fluoxetina, a maioria destes pacientes utilizam estes medicamentos em associação e/ou associado ao Clonazepam.

Foram identificados também medicamentos como: Escitalopram, Amitripitilna e Calman (calmante leve). Ademais, apenas 3 destes pacientes fazem acompanhamento psicoterápico e são os mesmos que continuaram o tratamento e evoluíram positivamente. Outro transtorno que se destaca é o Transtorno Bipolar, classificado como F31.1 no CID 10, no qual foi encontrado 7 pacientes do total (110) com esta patologia $(6,3 \%)$. Destes 7 , foi prescrito para 4 pacientes o Carbolitium (Estabilizador de Humor), 3 Quetiapina, 2 Amitripitilina, 2 Biperideno, 2 Ácido Valproico, 1 Risperidona e 1 Alpazolam (Benzodiazepínicos), visto que, a maioria desses pacientes fazem uso da politerapia. Por conseguinte, 4 pacientes dos 7 supracitados continuaram o tratamento e permanecem estáveis, destaca-se que destes, 2 precisaram da Assistência Social. Foi visto que 7 pacientes dos 110, com Distúrbio Intelectual (DI), antigo Retardo Mental no DSM IV, (6,3\%), classificado como F71 no CID 10 . Os medicamentos mais utilizados foram: Risperidona (3 pacientes) e Promitazina, anti-histamínico, (2 pacientes), além disso outros medicamentos como: Biperideno, Neuleptin, Haldol e Fluoxetina, foram utilizados como opção terapêutica. Dentre os pacientes com esta patologia apenas 2 continuaram o tratamento e permanecem estáveis e apenas 1 faz acompanhamento psicoterápico.

Outro dado observado foi uma menor prevalência do transtorno Misto de Ansiedade e Depressão, classificada como F41.2 no CID 10, com um total de 5 pacientes sendo o medicamento mais utilizado o Clonazepam, entretanto foi relatado também o uso de Sertralina (inibidores da recaptação da serotonina), Fluoxetina e Imipramina (antidepressivo tricíclico), na qual apenas 2 destes 5 pacientes continuaram o tratamento. Além disso, há um baixo número de diagnóstico de Autismo, classificada como F 84.0 no CID 10, com apenas 3 pacientes em tratamento e sendo contínuo, ou seja, todos os pacientes com Autismo tratados fazem retornos contínuos (a cada 3 meses) e se encontram estáveis. Por fim, destaca-se também, outras patologias menos prevalentes, com ' $n$ ' entre 1 e 2, tais como: Distúrbios da atividade de atenção (F90 no CID 10), Epilepsia (G40 no CID 10), Reação Aguda ao Stress (F43.0 no Cid 10), Transtorno de Pânico (F41 no CID 10), Transtorno Obsessivo Compulsivo (F42 no CID 10), Transtorno de Personalidade (F60 no Cid 10), automutilação (Z91.5 no CID 10), entre outras.

\section{DISCUSSÃO}

Com base nos resultados apresentados, podemos perceber uma desvalorização das doenças psíquicas como um todo, visto que o abandono do tratamento é muito recorrente, principalmente nas doenças consideradas mais leves e consequentemente negligenciadas. Exemplos destas são: o Transtorno de Ansiedade Generalizada (TAG) e o Transtorno Depressivo Maior (TDM), recorrente ou moderado. Isto pode ser percebido pela falta de adesão a terapia pois não tiveram consulta de retorno. No presente estudo verificou-se que a maioria dos pacientes (60\%) são do sexo feminino e $67 \%$ são maiores que 25 anos.
Tais dados vão de encontro ao relatado na literatura, que aponta como fatores predisponentes ao maior adoecimento no sexo feminino tendência a um estresse maior como jornada de trabalho e renda inferior, maternidade, maior exposição a violência que se expressa como forma de medo, angustia e ansiedade e além disso eventos que atuariam no sistema endócrino aumentando o risco de doenças psiquiátricas(Costa, Branco, Vieira, Souza, \& Silva, 2019). Além disso, há uma variável interessante, que a população feminina por ter mais cuidado com a saúde e mais facilidade para identificar sinais e sintomas sejam mais diagnosticadas, então sua prevalência acaba sendo alta(Silva, et al., 2018). Entretanto mostra-se também, que a prevalência no sexo feminino é mais elevada desde a infância e adolescência (Lopes, et al., 2016). Já em relação a faixa etária, podese destacar algumas variáveis como: maior acúmulo de doenças crônicas, dificuldade econômicas, isolamento social, episódios de estresse durante a vida estado civil (divorciado, separado ou viúvo) e instabilidade financeira(Santos, Lovisi, Legay, \& Abelha, 2009). Observou-se ainda, compatibilidade com a literatura (Hiany, Vieira, Gusmão, \& Barbosa, 2018) e os dados apresentados neste estudo em relação aos principais diagnósticos encontrados na população adulta. Os transtornos mais prevalentes na amostra foram Transtorno Depressivo Maior (F32), Transtorno de Ansiedade Generalizada (F41) e Esquizofrenia (F20). O presente estudo, ainda permitiu estabelecer uma relação dentre as doenças psíquicas identificadas com a metodologia terapêutica utilizada em cada patologia, sendo apenas psicofármacos ou associada a Terapia Cognitiva Comportamental (TCC), entretanto para uma melhor adesão e eficácia do tratamento recomenda-se a terapia combinada. O Transtorno Depressivo Maior (TDM) é um episódio depressivo sem história de mania ou hipomania, podendo apresentar alteração no peso, atividade, sono, falta de energia, sentimento de culpa e pensamentos suicidas. Visto os dados colhidos, 19 pacientes têm Transtorno Depressivo Recorrente adicionado a 10 do Transtorno Depressivo Moderado, somando 29, ou seja, 26\% dos pacientes psiquiátricos tem TDM, comparado com o Brasil é uma taxa alta pois é visto cerca de $17 \%$.

Notou-se que apenas 9 pacientes do total $(n=29)$ fazem acompanhamento psicoterápico e todos eles tiveram uma evolução positiva ao tratamento, tornando a TCC uma intervenção psicossocial essencial para um bom resultado a terapia do paciente psiquiátrico, visto que ajuda o paciente na redução de recaídas, diminuição dos sintomas, o não abandono ao tratamento e ainda investiga e trata os gatilhos da pessoa que possam estar sendo a piora do paciente. $\mathrm{O}$ Transtorno de Ansiedade Generalizada (TAG) é visto como uma ansiedade e/ou preocupação excessiva com eventos ou atividades na maior parte do tempo pelo menos durante 6 meses. Epidemiologicamente, $0,6 \%$ dos pacientes psiquiátricos da no município em estudo, já no Brasil varia de 3\% a $8 \%$. Ainda segundo Kaplan (2017), a terapia mais recomendada para o Transtorno de Ansiedade Generalizada é uma combinação em: Terapia Cognitivo Comportamental (TCC), medidas de apoio e psicoterapia(Sadock, Sadock, \& Ruiz, 2017). Como já citado, apenas 3 pacientes do total $(n=18)$ diagnosticados com TAG fazem acompanhamento psicoterápico e são os mesmos que deram continuidade a terapia mostrando a importância da terapia a estes pacientes que muitas vezes não buscam o tratamento correto ou não dão continuidade ao 
tratamento por banalizarem a patologia, principalmente se o caso de sintomas for leve. Além disso, a TCC irá ajudar o paciente a lidar com os sintomas frequentes da TAG como: agitação, insônia, nervosismo e preocupação, que orientará exercícios de meditação, atividades físicas e aconselhamentos (Fernandes, 2020). Neste estudo tiveram 12 pacientes diagnosticados com esquizofrenia do total de 110 caracterizando $10,9 \%$ desta população comparando com o Brasil que teve a prevalência de 0,3 a 2,4\%(Sadock, Sadock, \& Ruiz, 2017). A metodologia farmacológica recomendada em geral são antipsicóticos sendo típico ou atípico e onde todos têm uma característica em comum que é antagonizar os receptores de dopamina pós-sinápticos no cérebro. Entretanto deve-se ter muito cuidado com os efeitos colaterais no paciente que varia de sedação, efeitos anticolinérgicos até síndrome extrapiramidal. Destaca-se, a falta de adesão ao tratamento por ser a longo prazo e por precisar de ajuda familiar e/ou cuidador pois estes pacientes necessitam de auxílio para ingestão de medicamentos, atividade de vida diária além do apoio e suporte a eles. Neste estudo, 10 pacientes esquizofrênicos fazem uso da Politerapia (casos mais graves) com Depakene e/ou clonazepam. Foi visto também tratamento com Risperidona ou Biperidona, Quetiapina e Haldol sendo equivalente a estudos encontrados.

\section{CONCLUSÃO}

Como apresentado, nota-se a importância de melhor acurácia e empenho no diagnóstico precoce dos transtornos mentais. Notou-se, uma baixa adesão a intervenções psicossociais somada a terapia medicamentosa o que prejudica diretamente no sucesso do tratamento. O presente estudo evidencia a necessidade de medidas preventivas como redução de pontos de gatilho e do estresse na população, além de uma valorização maior do tema, para promoção da saúde mental e sua assistência.

\section{REFERÊNCIAS}

Amaral, C., Torrenté, M., Torrenté, M., \& Moreira, C. (2018). Apoio matricial em Saúde Mental na atenção básica: efeitos na compreensão e manejo por parte de agentes comunitários de saúde. Interface, 22(66). doi:https://doi.org/10.1590/180757622017.0473

Basso, L., \& Wainer, R. (2011). Luto e perdas repentinas: contribuições da Terapia Cognitivo-Comportamental. Rev. bras.ter. cogn., 7(1).
Costa, C., Branco, J., Vieira, I., Souza, L., \& Silva, R. (2019). Prevalência de ansiedade e fatores associados em adultos. $J$. bras. psiquiatr., 68(2). doi:https://doi.org/10.1590/0047-208500 0000232

Fernandes, L. (2020). Transtorno de Ansiedade Generalizada (TAG): Uma Breve Análise. Revista Farol, 10(10).

Gomes, G. (2019). Sintomas depressivos e fatores associados em idosos brasileiros. Universidade Federal de Pernambuco, Mestrado em Gerontologia. Recife: UFPE.

Hiany, N., Vieira, M., Gusmão, R., \& Barbosa, S. (2018). Perfil Epidemiológico dos Transtornos Mentais na População Adulta no Brasil: uma revisão integrativa. 86(24). doi: https://doi.org/ 10.31011/reaid-2018-v.86-n.24-art.676

Lopes, C., Abreu, G., Santos, D., Menezes, P., Carvalho, K., Cunha, C.,... Szklo, M. (2016). ERICA: prevalência de transtornos mentais comuns em adolescentes brasileiros. Rev. Saúde Pública, 50(1). doi:https://doi.org/10.1590/s01518-8787.201 6050006690

OMS, O. (2019). Saúde Mental. Organização Mundial da Saúde. Acesso em 11 de 11 de 2020, disponível em https://www.paho. org/bra/index.php?option=com_joomlabook\&view=topic\&id $=2$ 10

Sadock, B., Sadock, V., \& Ruiz, P. (2017). Compêndio de psiquiatria : ciência do comportamento e psiquiatria clínica. Porto Alegre: Artmed.

Santos, S., Lovisi, G., Legay, L., \& Abelha, 1. (2009). Prevalência de transtornos mentais nas tentativas de suicídio em um hospital de emergência no Rio de Janeiro, Brasil. Cad. Saúde Pública, 25(9), 2064-2074. doi:https://doi.org/10.1590/S0102-311X2009 000900020

Silva, P., Rocha, S., Santos, L., Santos, C., Amorim, C., \& Vilela, A. (2018). Prevalência de transtornos mentais comuns e fatores associados entre idosos de um município do Brasil. Ciênc. saúde colet., 23(2). doi:https://doi.org/10.1590/1413-81232018232. 12852016

Smolen, J., \& Araújo, E. (2017). Raça/cor da pele e transtornos mentais no Brasil: uma revisão sistemática. Ciênc. saúde colet, 22(12). doi:https://doi.org/10.1590/1413-812320172212. 1978 2016

Soares, W., Coutinho, J., Alves, G., Moura, K., Aquino, D., Ribeiro, N., Souza, I. (2021). Contextualização da saúde mental no brasil associada ao tratamento farmacológico com antipsicóticos. Brazilian Journal of Health Review, 4(1), 1997-2010. 